Proc. Estonian Acad. Sci. Phys. Math., 2007, 56, 4, 347-358

\title{
Observability of a class of linear dynamic infinite systems on time scales
}

\author{
Dorota Mozyrska and Zbigniew Bartosiewicz \\ Faculty of Computer Science, Białystok Technical University, Wiejska 45A, 15-351 Białystok, \\ Poland; admoz@w.tkb.pl, bartos@pb.bialystok.pl \\ Received 23 February 2007, in revised form 4 July 2007

\begin{abstract}
Linear dynamic systems with output, evolving on the space $\mathbb{R}^{\infty}$ of infinite sequences, are studied. They are described by infinite systems of $\Delta$-differential linear equations with row-finite matrices, for which time belongs to an arbitrary time scale. Such systems generalize discrete-time and continuous-time row-finite systems on $\mathbb{R}^{\infty}$, studied earlier. Necessary and sufficient conditions on observability of such systems are given. Formal
\end{abstract} \\ polynomial series on time scales are introduced.
}

Key words: infinite linear $\Delta$-differential system, time scale, observability.

\section{INTRODUCTION}

The theory of dynamical systems on time scales unifies theories of continuoustime and discrete-time systems. It also allows considering a dynamical system with time, which is partly continuous and partly discrete. Observability of finitedimensional, stationary, linear systems on time scales was studied in $\left[{ }^{1}\right]$. It was shown that the standard Kalman criterion of observability holds for systems on an arbitrary time scale. We prove here a similar result for a class of infinite systems of linear differential equations with output. The systems are described by infinite matrices whose rows have only finitely many nonzero elements and the state space consists of all infinite sequences. In $\left[^{2}\right]$ we studied observability of discrete-time systems of this type and proved an extension of the Kalman criterion to infinite systems. As its consequence the following characterization of observability was derived: the system is observable if and only if every state variable can be expressed as a finite linear combination of the output and its time-shifts. In $\left[{ }^{3}\right]$ we showed 
that the same holds for continuous-time infinite systems, with time-shifts replaced by time-derivatives. However, in that case, to have existence and uniqueness of solutions, we were forced to consider formal solutions defined by formal power series. As we consider here a more general situation of arbitrary time case, the difficulties that appeared in the continuous-time case must inevitably show up also in this paper. For this purpose we introduce formal polynomial series on time scales, formal solutions described by such series, and formal exponential matrices. Once we obtain the Kalman criterion of observability, we can restate all the results of $\left[{ }^{2}\right]$ and $\left[{ }^{3}\right]$, as the time scale becomes no longer essential.

The systems we study here are infinite-dimensional, but they are far from the mainstream of the infinite-dimensional system theory, which consists mainly of two areas: systems described by partial differential equations and systems on Banach or Hilbert spaces (see, e.g., $\left.\left[{ }^{4}\right]\right)$. The space of all real sequences is neither a Banach space nor a Hilbert space. It has the structure of a Fréchet space, but the theory of systems on Fréchet spaces is not developed yet. Most of the examples of infinite-dimensional systems lead to systems on Banach spaces, but the systems that we study here can also serve as models of certain dynamics. For example, the infinite extension of a finite-dimensional control system leads to a system whose state space consists of all infinite sequences. Such a system appears also as an effect of dicretization of the heat equation. Moreover, during this process we can obtain systems with time belonging to different time scales. If we discretize all the variables, we obtain a system with the time scale $\mathbb{Z}$, while discretizing only the space variables, we end up with the time scale $\mathbb{R}$. The results concerning observability obtained in this paper coincide in principal with the results obtained for systems on Banach spaces, but cannot be deduced from them. Moreover, since we study systems on concrete spaces, we can make the most of the structure of such systems. For example, the row-finiteness of the matrices that describe the systems allows for a nice characterization of observability as a possibility of expressing the state variables as linear combinations of finitely many $\Delta$-derivatives of the output functions.

\section{INFINITE MATRICES}

Let $K$ be a nonempty countable set. Consider the countable product $\mathbb{R}^{K}=$ $\prod_{k \in K} \mathbb{R}$ as the set of all functions $K \rightarrow \mathbb{R}$. If $K=\mathbb{N}$, then $\mathbb{R}^{\mathbb{N}}$ is the linear space of all infinite sequences of real numbers represented by infinite columns $x=\left(x_{1}, \ldots, x_{i}, \ldots\right)^{T}, x_{i} \in \mathbb{R}, i \in \mathbb{N}$. The space $\mathbb{R}^{\mathbb{N}}$ with the product topology (the Tikhonov topology) is metrizable. However, there is no norm for this topology, so $\mathbb{R}^{\mathbb{N}}$ is not a Banach space.

Recall that a linear topological space is called a Fréchet space if it is metrizable, complete and locally convex. Note that $\mathbb{R}^{\mathbb{N}}$ is a Fréchet space $\left[{ }^{5}\right]$.

We have the following: 
Proposition 2.1. $\left[{ }^{5}\right]$ A function $f: \mathbb{R}^{\mathbb{N}} \rightarrow \mathbb{R}$ is linear and continuous iff there is a finite set $S \subset \mathbb{N}$ and a set of real numbers $\left\{a_{i}\right\}_{i \in S}$ such that for all $x \in \mathbb{R}^{\mathbb{N}}$ : $f(x)=\sum_{i \in S} a_{i} x_{i}$, where $x_{i}=x(i)$.

Let $K=\mathbb{N} \times \mathbb{N}$. Then each element $A \in \mathbb{R}^{\mathbb{N} \times \mathbb{N}}, A: \mathbb{N} \times \mathbb{N} \ni(i, j) \mapsto a_{i j} \in \mathbb{R}$, is called an infinite matrix. We will denote it in the standard way $A=\left(a_{i j}\right)_{i, j \in \mathbb{N}}$. By $I=\left(\delta_{i j}\right)_{i, j \in \mathbb{N}}$, where $\delta_{i j}=0$ for $i \neq j, \delta_{i i}=1$, we denote the identity matrix in $\mathbb{R}^{\mathbb{N} \times \mathbb{N}}$. The set $\mathbb{R}^{\mathbb{N} \times \mathbb{N}}$ of all infinite matrices is a linear space over $\mathbb{R}$ with the standard operations.

Now let $A=\left(a_{i j}\right), B=\left(b_{i j}\right)$ be infinite matrices. Then the product $A B=\left(c_{i k}\right)_{i, k \in \mathbb{N}}$ is well defined if the series $c_{i k}=\sum_{j \in \mathbb{N}} a_{i j} b_{j k}$ is convergent for each $(i, k) \in \mathbb{N} \times \mathbb{N}$.

Definition 2.2. We say that $A=\left(a_{i j}\right)_{i, j \in \mathbb{N}}$ is

a) row-finite if for each $i \in \mathbb{N}$ there is $\alpha(i) \in \mathbb{N}: a_{i j}=0$ for $j>\alpha(i)$,

b) column-finite if $A^{T}$ is row-finite,

c) lower-diagonal if $a_{i j}=0$ for $j>i$,

d) upper-diagonal if $a_{i j}=0$ for $j<i$.

Of course, a lower-diagonal matrix is a particular case of a row-finite matrix. The identity matrix $I$ is row-finite, column-finite, and lower(upper)-diagonal.

\section{Proposition 2.3.}

1. The set of all infinite row-finite matrices forms an algebra over $\mathbb{R}$ with a unitthe identity matrix I.

2. The set of all infinite column-finite matrices forms an algebra over $\mathbb{R}$ with a unit.

Remark 2.4. The associativity of multiplication is the most essential property for row-finite (column-finite) matrices. It does not hold for all infinite matrices, however.

From Proposition 2.3 we get that if $A$ is a row-finite matrix, then all powers $A^{k}, k \in \mathbb{N} \cup\{0\}$, have the same property. Let us denote by $\left(A^{k}\right)_{i j}$ the element at the $i$ th row and $j$ th column of matrix $A^{k}$. Now we recall the definition of the matrix exponential of an infinite matrix. It was discussed in $\left[{ }^{6}\right]$.

Definition 2.5. $\left[{ }^{7}\right]$ Let $A=\left(a_{i j}\right)_{i \in \mathbb{N}, j \in \mathbb{N}}$ be an infinite matrix and suppose that there is $r>0$ such that for each $i, j \in \mathbb{N}$ the power series $\sum_{k=0}^{\infty} \frac{t^{k}}{k !}\left(A^{k}\right)_{i j}$ has the radius of convergence greater than or equal to $r>0$. Then we define the matrix $\mathrm{e}^{t A}$ by $\left(\mathrm{e}^{t A}\right)_{i j}=\sum_{k=0}^{\infty} \frac{t^{k}}{k !}\left(A^{k}\right)_{i j}$.

Example 2.6. Let $A$ be the upper-diagonal matrix of the following form:

$$
A=\left(\begin{array}{cccccc}
0 & 1 & 0 & 0 & 0 & \ldots \\
0 & 0 & 1 & 0 & 0 & \ldots \\
0 & 0 & 0 & 1 & 0 & \ldots \\
\vdots & \vdots & \vdots & \ddots & \ddots & \ddots
\end{array}\right)
$$


Then the exponential matrix exists and is equal to

$$
\mathrm{e}^{t A}=\left(\begin{array}{ccccc}
1 & t & \frac{t^{2}}{2 !} & \frac{t^{3}}{3 !} & \cdots \\
0 & 1 & t & \frac{t^{2}}{2 !} & \cdots \\
0 & 0 & 1 & t & \cdots \\
\vdots & \vdots & \ddots & \ddots & \ddots
\end{array}\right)
$$

Remark 2.7. If an infinite matrix $A$ is a lower-diagonal matrix, then the exponential matrix $\mathrm{e}^{A t}=I+A t+A^{2} \frac{t^{2}}{2 !}+\cdots$ is well defined [ $\left.{ }^{8}\right]$.

In Section 4 we shall give the definition of formal exponential matrix on an arbitrary time scale.

\section{CALCULUS ON TIME SCALES}

In this section we give a short collection of the most important facts from the calculus on time scales. For further reading we refer to $\left[{ }^{9-11}\right]$.

By a time scale, denoted here by $\mathbb{T}$, we mean a nonempty closed subset of $\mathbb{R}$. As the theory of time scale gives the way to unify continuous and discrete analysis, the standard cases of time scales are the following: $\mathbb{T}=\mathbb{R}, \mathbb{T}=\mathbb{Z}$ or $\mathbb{T}=h \mathbb{Z}, h>0$.

For $t \in \mathbb{T}$ we define the forward jump operator $\sigma$ and the graininess $\mu$ by:

a) $\sigma(t)=\inf \{s \in \mathbb{T}: s>t\}$ and $\sigma(\sup \mathbb{T})=\sup \mathbb{T}$ if $\sup \mathbb{T}<+\infty$;

b) $\mu(t)=\sigma(t)-t$.

Moreover, we have the backward operator $\rho$ defined by: $\rho(t)=\sup \{s \in \mathbb{T}$ : $s<t\}$ and $\rho(\inf \mathbb{T})=\inf \mathbb{T}$ if inf $\mathbb{T}>-\infty$. In the continuous-time case, when $\mathbb{T}=\mathbb{R}$, we have that for all $t \in \mathbb{R}: \sigma(t)=\rho(t)=t$ and $\mu(t)=0$. In the discrete-time case, for each $t \in \mathbb{T}=\mathbb{Z}: \sigma(t)=t+1, \rho(t)=t-1, \mu(t)=1$.

Additionally we define the set $\mathbb{T}^{k}$ as: $\mathbb{T}^{k}:=\mathbb{T} \backslash(\rho(\sup \mathbb{T})$, sup $\mathbb{T}]$ if $\sup \mathbb{T}<\infty$ and $\mathbb{T}^{k}=\mathbb{T}$ if $\sup \mathbb{T}=\infty$.

For a function $f: \mathbb{T} \rightarrow \mathbb{R}$ we define the delta derivative of $f$ at $t \in \mathbb{T}^{k}$, denoted by $f^{\Delta}(t)$, to be the number, if it exists, with the property that for all $\varepsilon>0$ there is a neighbourhood $U \subset \mathbb{T}$ of $t$ such that for all $s \in U$ it holds that

$$
\left|f(\sigma(t))-f(s)-f^{\Delta}(t)(\sigma(t)-s)\right| \leq \varepsilon|\sigma(t)-s| .
$$

Moreover, we say that the function $f$ is delta differentiable on $\mathbb{T}^{k}$ provided $f^{\Delta}(t)$ exists for all $t \in \mathbb{T}^{k}$.

\section{Example 3.1.}

1. Let $\mathbb{T}=\mathbb{R}$, then $f^{\Delta}(t)=f^{\prime}(t)$ and $f$ is delta differentiable iff it is differentiable in the ordinary sense.

2. Let $\mathbb{T}=\mathbb{Z}$, then $f^{\Delta}(t)=f(t+1)-f(t)$ and it always exists.

3. Let $\mathbb{T}$ be any time scale, then the delta derivative of $t^{2}$ is $t+\sigma(t)$ and $(t+\sigma(t))^{\Delta}$ may not exist. 
A function $f: \mathbb{T} \rightarrow \mathbb{R}$ is called regulated if its right-side limits exist (finite) at all points $t \in \mathbb{T}$ with $\sigma(t)=t$ and its left-side limits exist (finite) at all points $t \in \mathbb{T}$ with $\rho(t)=t$. A regulated function $f$ has a pre-antiderivative, i.e. a continuous $F$ with $F^{\Delta}=f$ on a set $D$ where $\mathbb{T} \backslash D$ is countable and does not contain any points $t$ with $\sigma(t)>t$. Then the Cauchy integral can be defined as: $\int_{a}^{b} f(t) \Delta t=F(b)-F(a)$.

\section{Example 3.2.}

1. Let $\mathbb{T}=\mathbb{R}$, then $\int_{a}^{b} f(t) \Delta t$ is equal to the usual Riemann integral.

2. Let $\mathbb{T}=\mathbb{Z}$, then $\int_{a}^{b} f(t) \Delta t=\sum_{t=a}^{b-1} f(t)$.

Let $\mathbb{N}_{0}$ mean the set of natural numbers with zero. For $t \in \mathbb{R}$ and $k \in \mathbb{N}$ we define

$$
t^{(0)}:=1 \text { and } t^{(k)}:=t(t-1) \cdots(t-k+1) .
$$

If $t \in \mathbb{Z}$ and $k \geq t+1, k \in \mathbb{N}$, then $t^{(k)}=0$.

Let $\mathbb{T}$ be a time scale. We use the functions $h_{k}: \mathbb{T} \times \mathbb{T} \longrightarrow \mathbb{R}, k \in \mathbb{N} \cup\{0\}$, defined recursively as follows $\left[{ }^{9,10}\right]$ :

$$
h_{0}\left(t, t_{0}\right) \equiv 1 \text { and } h_{k+1}\left(t, t_{0}\right)=\int_{t_{0}}^{t} h_{k}\left(\tau, t_{0}\right) \Delta \tau .
$$

Let $h_{k}^{\Delta}\left(t, t_{0}\right)$ denote, for each fixed $t_{0}$, the derivative of $h_{k}\left(t, t_{0}\right)$ with respect to $t$. Then, for $t \in \mathbb{T}^{\kappa}$,

$$
h_{0}^{\Delta}\left(t, t_{0}\right) \equiv 0 \text { and } h_{k}^{\Delta}\left(t, t_{0}\right)=h_{k-1}\left(t, t_{0}\right), k \geq 1 .
$$

In the case $\mathbb{T}=\mathbb{Z}$ we have that $h_{k}\left(t, t_{0}\right)=\frac{\left(t-t_{0}\right)^{(k)}}{k !}$ for all $t, t_{0} \in \mathbb{Z}$ and $k \in \mathbb{N}$. Moreover, if $\mathbb{T}=\mathbb{R}$, then $h_{k}\left(t, t_{0}\right)=\frac{\left(t-t_{0}\right)^{k}}{k !}$, and it gives a universal upper bound in the following way:

Theorem 3.3. $\left[{ }^{11}\right]$ Let $k \in \mathbb{N} \cup\{0\}$. Then for all $t \geq t_{0}$ it holds that

$$
0 \leq h_{k}\left(t, t_{0}\right) \leq \frac{\left(t-t_{0}\right)^{k}}{k !} .
$$

\section{FORMAL POLYNOMIAL SERIES ON TIME SCALES}

Let $X$ be a linear space (over $\mathbb{R}$ ). Then by $X^{\mathbb{N}_{0}}$ we denote the set of all infinite sequences of elements from $X$. Hence $X^{\mathbb{N}_{0}}=\left\{a=\left(a_{k}\right)_{k \in \mathbb{N}_{0}}: a_{k} \in X\right\}$. We define the addition of two sequences by $\left(a_{k}\right)+\left(b_{k}\right)=\left(a_{k}+b_{k}\right)$ and the multiplication by scalars by $\alpha\left(a_{k}\right)=\left(\alpha a_{k}\right), \alpha \in \mathbb{R}$. Then $X^{\mathbb{N}_{0}}$ with these operations on sequences becomes a linear space over $\mathbb{R}$.

Remark 4.1. If in $X$ we have a metric $d$, then in the space $X^{\mathbb{N}_{0}}$ we can introduce a metric defined by $\rho(a, b)=\sum_{k=0}^{\infty} \frac{d\left(a_{k}, b_{k}\right)}{1+d\left(a_{k}, b_{k}\right)}$, where $a, b \in X^{\mathbb{N}_{0}}$. 
In $X^{\mathbb{N}_{0}}$ we can define the shift operator by $D\left(a_{0}, a_{1}, \ldots\right)=\left(a_{1}, a_{2}, \ldots\right)$. This operation is $\mathbb{R}$-linear, i.e. $D(\alpha a+\beta b)=\alpha D(a)+\beta D(b)$, for $\alpha, \beta \in \mathbb{R}$ and $a, b \in X^{\mathbb{N}_{0}}$.

A formal power series in a real variable $t$ with coefficients from a linear space $X$ over $\mathbb{R}$ has the form of an infinite sum of monomials: $\sum_{k=0}^{\infty} a_{k} t^{k}$, where for every $k \in \mathbb{N}_{0}, a_{k} \in X$. Operations like sum, and multiplying by scalars are defined on series in a similar way as on sequences. Shifting of sequences from $X^{\mathbb{N}_{0}}$ is better represented as an operation on polynomial series given by $\sum_{k=0}^{\infty} a_{k} \frac{t^{k}}{k !}$, where $a=\left(a_{0}, a_{1}, \ldots\right) \in X^{\mathbb{N}_{0}}$. Then the operation of formal differentiation of the series corresponds to the action of the shift operator on the sequence of coefficients of the series.

To do the same on an arbitrary time scale $\mathbb{T}$, we use the generalized polynomials, defined by (3.2), in the definition of formal polynomial series on time scales.

Definition 4.2. Let $\mathbb{T}$ be a time scale and $t, t_{0} \in \mathbb{T}$. Let $X$ be a linear space over $\mathbb{R}$. By a formal polynomial series (on $\mathbb{T}$, centred at $t_{0}$ ) over $X$ we mean a formal expression

$$
\mathcal{S}_{a}^{t_{0}}=\sum_{k=0}^{\infty} a_{k} h_{k}\left(t, t_{0}\right),
$$

where $a=\left(a_{k}\right)_{k \in \mathbb{N}_{0}} \in X^{\mathbb{N}_{0}}$. The sequence $a$ is called the corresponding sequence of $\mathcal{S}_{a}^{t_{0}}$. Two formal polynomial series on the time scale $\mathbb{T}$, at the point $t_{0} \in \mathbb{T}$, are equal if their corresponding sequences are equal, i.e. $\mathcal{S}_{a}^{t_{0}}=\mathcal{S}_{b}^{t_{0}} \Longleftrightarrow \forall\left(k \in \mathbb{N}_{0}\right)$ : $a_{k}=b_{k}$. The set of all formal polynomial series (on $\mathbb{T}$, centred at $t_{0}$ ) over $X$ are denoted by $X\left[\left[t, t_{0}\right]\right]$.

Similarly as sequences from $X^{\mathbb{N}_{0}}$, the series like (4.1) can be added and multiplied by scalars in the following way:

$$
\begin{gathered}
\sum_{k=0}^{\infty} a_{k} h_{k}\left(t, t_{0}\right)+\sum_{k=0}^{\infty} b_{k} h_{k}\left(t, t_{0}\right)=\sum_{k=0}^{\infty}\left(a_{k}+b_{k}\right) h_{k}\left(t, t_{0}\right) ; \\
\alpha\left(\sum_{k=0}^{\infty} a_{k} h_{k}\left(t, t_{0}\right)\right)=\sum_{k=0}^{\infty}\left(\alpha a_{k}\right) h_{k}\left(t, t_{0}\right), \alpha \in \mathbb{R} .
\end{gathered}
$$

Proposition 4.3. The set of all formal polynomial series (on $\mathbb{T}$, centred at $t_{0}$ ) over the linear (metrizable) space $X$ is a linear (metrizable) space (over $\mathbb{R}$ ) with the addition and the multiplication by scalars defined by (4.2) and (4.3).

Given a formal polynomial series $\mathcal{S}_{a}^{t_{0}}=\sum_{k=0}^{\infty} a_{k} h_{k}\left(t, t_{0}\right)$, we define its formal $\Delta$-derivative by

$$
\left(\mathcal{S}_{a}^{t_{0}}\right)^{\Delta}=\sum_{k=0}^{\infty} a_{k+1} h_{k}\left(t, t_{0}\right) .
$$


Hence the formal $\Delta$-derivative of the series $\mathcal{S}_{a}^{t_{0}}$ is the new formal polynomial series (on $\mathbb{T}$, centred at $\left.t_{0}\right)$ with the shifted corresponding sequence $\left(a_{1}, a_{2}, \ldots\right) \in$ $X^{\mathbb{N}_{0}}$.

Remark 4.4. If we fix the value of $t \in \mathbb{T}$, then the expression $\mathcal{S}_{a}^{t_{0}}$, with $a \in X^{\mathbb{N}_{0}}$, becomes the series of elements from the space $X$. As we do not assume that $X$ is a metric space, hence, in general, we cannot consider convergence of the series of elements. But if $X$ is metrizable or if the sum is finite, we can investigate the convergence.

By Theorem 3.3, we have the following:

Corollary 4.5. Let $\mathbb{T}$ be any time scale and let $t_{0} \in \mathbb{T}$. Then the convergence of the series $\sum_{k=0}^{\infty} a_{k} \frac{\left(t-t_{0}\right)^{k}}{k !}$ implies the convergence of the series with the same corresponding sequence $\sum_{k=0}^{\infty} a_{k} h_{k}\left(t, t_{0}\right)$ on $\mathbb{T}$.

In particular, when $\mathbb{T}=\mathbb{Z}$, for every $t \in \mathbb{Z}$, the series $\sum_{k=0}^{\infty} a_{k} h_{k}\left(t, t_{0}\right)$ reduces to the finite sum $\sum_{k=0}^{t-t_{0}} a_{k} h_{k}\left(t, t_{0}\right)=\sum_{k=0}^{t-t_{0}} a_{k}\left(\begin{array}{c}t-t_{0} \\ k\end{array}\right)$. If, additionally, for each $k \in \mathbb{N}_{0}: a_{k}=c^{k}, c \in \mathbb{R}$, then $\sum_{k=0}^{\infty} c^{k} h_{k}\left(t, t_{0}\right)=(1+c)^{t-t_{0}}, t \geq t_{0}$.

Let $P$ be the ring of infinite row-finite matrices. Then $P\left[\left[t, t_{0}\right]\right]$ is the set of all formal polynomial series (on $\mathbb{T}$, centred at $t_{0}$ ) with corresponding matrix sequences. Let us take $X=\mathbb{R}^{\mathbb{N}}$. Then for $a \in(X)^{\mathbb{N}_{0}}$ the formal polynomial series (on $\mathbb{T}$, centred at $t_{0}$ ) $\mathcal{S}_{a}^{t_{0}}$ has a vector form. So we write

$$
\mathcal{S}_{a}^{t_{0}}=\left(\sum_{k=0}^{\infty}\left(a_{k}\right)_{1} h_{k}\left(t, t_{0}\right), \ldots, \sum_{k=0}^{\infty}\left(a_{k}\right)_{i} h_{k}\left(t, t_{0}\right), \ldots\right)^{T}
$$

or as the family of scalar series: $\mathcal{S}_{a}^{t_{0}}=\left\{\sum_{k=0}^{\infty}\left(a_{k}\right)_{i} h_{k}\left(t, t_{0}\right)\right\}_{i \in \mathbb{N}}$.

Definition 4.6. Let $P$ be the ring of infinite row-finite matrices and $X=\mathbb{R}^{\mathbb{N}}$. Then for $\mathcal{S}_{\left(A_{0}, A_{1}, \ldots\right)}^{t_{0}} \in P\left[\left[t, t_{0}\right]\right]$ and $x_{0} \in X$ we define $\mathcal{S}_{\left(A_{0}, A_{1}, \ldots\right)}^{t_{0}} x_{0}:=$ $\mathcal{S}_{\left(A_{0} x_{0}, A_{1} x_{0}, \ldots\right)}^{t_{0}}=\sum_{k=0}^{\infty}\left(A_{k} x_{0}\right) h_{k}\left(t, t_{0}\right)$ as the formal scale-power series from $X\left[\left[t, t_{0}\right]\right]$.

Definition 4.7. Let $P$ be the ring of infinite row-finite matrices. Let $A \in P$. Then $\left(A^{0}, A, A^{2}, \ldots\right) \in P^{\mathbb{N}_{0}}$. Then we define the formal matrix exponent of $A$ (at $\left.t_{0}\right)$ as the formal polynomial series on $\mathbb{T}$ :

$$
\mathcal{E}_{A}^{t_{0}}:=\sum_{k=0}^{\infty} A^{k} h_{k}\left(t, t_{0}\right)
$$

and for $x_{0} \in \mathbb{R}^{\mathbb{N}}$ we write

$$
\mathcal{E}_{A}^{t_{0}} x_{0}=\sum_{k=0}^{\infty}\left(A^{k} x_{0}\right) h_{k}\left(t, t_{0}\right) .
$$


As in the expression (4.5) we have values of the functions $h_{k}\left(\cdot, t_{0}\right)$, the formal exponent depends on the time scale. If $\mathbb{T}=\mathbb{R}$ and $A$ is lower-diagonal, then the series $\mathcal{E}_{A}^{t_{0}}$ is the matrix of scalar series and this coincides with Definition 2.5.

If $\mathbb{T}=\mathbb{Z}$, then $\left(\mathcal{E}_{A}^{t_{0}}\right)_{i j}=\sum_{k=0}^{t-t_{0}}\left(\begin{array}{c}t-t_{0} \\ k\end{array}\right) A^{k}=(I+A)^{t-t_{0}}$ for fixed $t \in \mathbb{T}$. Hence $\mathcal{E}_{A}^{t_{0}}=(I+A)^{t-t_{0}}$ and has the same form as in the finite-dimensional case.

Proposition 4.8. Let $t, t_{0} \in \mathbb{T}$. Then $\left(\mathcal{E}_{A}^{t_{0}}\right)^{\Delta}=A \mathcal{E}_{A}^{t_{0}}$.

Corollary 4.9. Let $t, t_{0} \in \mathbb{T}$. Then $\left(\mathcal{E}_{A}^{t_{0}} x_{0}\right)^{\Delta}=A \mathcal{E}_{A}^{t_{0}} x_{0}$.

Proof. Let us observe that

$$
\left(\mathcal{E}_{A}^{t_{0}} x_{0}\right)_{i}^{\Delta}=\sum_{k=0}^{\infty}\left(A^{k+1}\right)_{i} x_{0} h_{k}\left(t, t_{0}\right)=\sum_{k=0}^{\infty} A_{i}\left(A^{k} x_{0}\right) h_{k}\left(t, t_{0}\right) .
$$

Then, as $A_{i}$ has only a finite number of elements different from zero, we have the following: $\left(\mathcal{E}_{A}^{t_{0}} x_{0}\right)_{i}^{\Delta}=A_{i}\left(\sum_{k=0}^{\infty}\left(A^{k} x_{0}\right) h_{k}\left(t, t_{0}\right)\right)$. Hence, for each $i \in \mathbb{N}$ it holds that $\left(\mathcal{E}_{A}^{t_{0}} x_{0}\right)_{i}^{\Delta}=\left(A \mathcal{E}_{A}^{t_{0}} x_{0}\right)_{i}$.

\section{INFINITE SYSTEMS OF $\Delta$-DIFFERENTIAL EQUATIONS}

We consider here linear systems of infinitely many $\Delta$-differential equations of the form

$$
x^{\Delta}(t)=A x(t),
$$

where $x(t) \in \mathbb{R}^{\mathbb{N}}$ and $A$ is a row-finite infinite real matrix (Definition 2.2).

Such systems may appear as infinite extensions of finite-dimensional control systems on time scales (in particular, continuous-time or discrete-time systems) of the form $y^{\Delta}(t)=F y(t)+G u(t)$, when we extend the state space adding new variables: $u$ and all its $\Delta$-derivatives $u^{[k]}$, for $k \in \mathbb{N}$.

Such systems are also related to infinite-dimensional systems described by partial differential equations. Let us consider a problem of discretization for such a system.

Example 5.1. The parabolic equation $\frac{\partial u}{\partial t}=\frac{\partial^{2} u}{\partial x^{2}}$ may be discretized with respect to the space variable $x$. Let $z_{k}(t)=u(t, k), k \in \mathbb{Z}$. If we replace $\frac{\partial^{2} u(t, x)}{\partial x^{2}}$ by $u(t, x+2)-2 x(t, x+1)+x(t, x)$, then the discretized system is given by the infinite number of differential equations:

$$
\dot{z}_{k}(t)=z_{k}(t)-2 z_{k+1}(t)+z_{k+2}(t), \quad k \in \mathbb{Z} .
$$

Observe that the equations are indexed by integers and not natural numbers as it was presented earlier. This leads to a row-finite matrix whose columns and 
rows are indexed by integers as well. One can also discretize the time variable replacing $\dot{z}_{k}(t)$ by $z_{k}(t+1)-z_{k}(t)$, i.e. by $z^{\Delta}(t)$, where the $\Delta$-derivative is taken on the time scale $\mathbb{Z}$. Other time scales can be used for time discretization as well (for example nonhomogeneous ones). Thus $z^{\Delta}(t)$ may mean different things, expressing different ways of discretization.

More examples of systems of differential equations described by infinite matrices can be found in $\left[{ }^{7}\right]$. Although such systems do not belong to the mainstream of the infinite-dimensional system theory, they pose interesting mathematical problems and have many important applications.

Let us consider now the initial value problem on $\mathbb{T}$

$$
x^{\Delta}(t)=A x(t), x\left(t_{0}\right)=x_{0} \in \mathbb{R}^{\mathbb{N}},
$$

where $A$ is a row-finite matrix, $t \in\left[t_{0}, \infty\right) \cap \mathbb{T}$, and $x \in \mathbb{R}^{\mathbb{N}}$. In the finitedimensional case the initial value problem $x^{\Delta}(t)=A x(t), x\left(t_{0}\right)=x_{0}$ has the unique forward solution (for $t>t_{0}$, where $t, t_{0} \in \mathbb{T}$ ) even if $A$ is not regressive $\left[{ }^{10}\right]$.

If $\mathbb{T}=\mathbb{N}$, then (5.2) is a discrete-time and infinite-dimensional initial value problem. Because $A^{k}, k \in \mathbb{N}_{0}$ is row-finite in case $A$ is row-finite, there is no difficulty with the existence and uniqueness of the forward solution. The solution, corresponding to the initial condition $x\left(t_{0}\right)=x_{0}$, is in the following form: $x(t)=(I+A)^{t-t_{0}} x_{0}$.

If $\mathbb{T}=\mathbb{R}$, the initial value problem (5.2) may have infinitely many smooth solutions and to get uniqueness we have considered, in $\left[{ }^{3}\right]$, formal solutions in the form of formal power series. Hence, similarly as in the continuous-time case, we shall use formal expressions for solutions of (5.2).

Proposition 5.2. Let $\mathbb{T}$ be any time scale and for $t_{0} \in \mathbb{T}: x\left(t_{0}\right)=x_{0} \in \mathbb{R}^{\mathbb{N}}$. Then the vector of formal polynomial series (on $\mathbb{T}$, centred at $t_{0}$ ) defined by (4.6) is the unique (formal) solution of the initial value problem (5.2).

Let $\Lambda$ be the system on $\mathbb{T}$ of the following form:

$$
\begin{gathered}
x^{\Delta}(t)=A x(t), \\
y(t)=C x(t),
\end{gathered}
$$

where $x(t) \in \mathbb{R}^{\mathbb{N}}$ and $y(t) \in \mathbb{R}^{J}$, where $J=\mathbb{N}$ or $J=p<\infty$. The matrices $A$ and $C$ are row-finite. In the case where $J=p$ the matrix $C$ has a finite number of rows but infinitely many columns. If $\mathbb{T}=\mathbb{N}$, then $\Lambda$ is a discrete-time system and, as we notice in the above discussion, there exists a unique (forward) solution of (5.3), and the output trajectory corresponding to the initial condition $x\left(t_{0}\right)=x_{0}$ has just the form $y(t)=C A^{t-t_{0}} x_{0}$. For any other time scale $\mathbb{T}$ we have formal output $\mathcal{Y}_{x_{0}}^{t_{0}}=\sum_{k=0}^{\infty} C A^{k} x_{0} h_{k}\left(t, t_{0}\right)$.

The initial condition $x\left(t_{0}\right)=x_{0}$ may be represented as a pair $\left(t_{0}, x_{0}\right) \in \mathbb{T} \times \mathbb{R}^{\mathbb{N}}$. Such a pair is called an event. 
Definition 5.3. We say that two events $\left(t_{0}, x_{1}\right)$ and $\left(t_{0}, x_{2}\right)$ are indistinguishable by the system $\Lambda$ if $\mathcal{Y}_{x^{1}}^{t_{0}}=\mathcal{Y}_{x^{2}}^{t_{0}}$. Otherwise, the events $\left(t_{0}, x_{1}\right)$ and $\left(t_{0}, x_{2}\right)$ are distinguishable. We say that the system $\Lambda$ is observable if for any two distinct points $x_{1}, x_{2} \in \mathbb{R}^{\mathbb{N}}$ there is $t_{0} \in \mathbb{T}$ such that the events $\left(t_{0}, x_{1}\right),\left(t_{0}, x_{2}\right)$ are distinguishable.

Proposition 5.4. The events $\left(t_{0}, x_{1}\right)$ and $\left(t_{0}, x_{2}\right)$ are indistinguishable iff for all $k \in \mathbb{N}_{0}: C A^{k} x^{1}=C A^{k} x^{2}$.

Proof. $\mathcal{Y}_{x^{1}}^{t_{0}}=\mathcal{Y}_{x^{2}}^{t_{0}} \Leftrightarrow \sum_{k=0}^{\infty} C A^{k} x^{1} h_{k}\left(t, t_{0}\right)=\sum_{k=0}^{\infty} C A^{k} x^{2} h_{k}\left(t, t_{0}\right) \Leftrightarrow \forall k \in$ $\mathbb{N}_{0}: C A^{k} x^{1}=C A^{k} x^{2}$.

Remark 5.5. If there is $t_{0} \in \mathbb{T}$ such that two events $\left(t_{0}, x_{1}\right),\left(t_{0}, x_{2}\right)$ are distinguishable, then it means that for all $t \in \mathbb{T}$ the events $\left(t, x_{1}\right),\left(t, x_{2}\right)$ are distinguishable.

Observe that the conditions on observability that were obtained for $\mathbb{T}=\mathbb{R}$, in $\left[{ }^{3}\right]$, are the same for any time scale $\mathbb{T}$. Hence we recall main propositions without repeating the proofs, which can be found in $\left.{ }^{2,3}\right]$.

Let $f: \mathbb{N} \times \mathbb{N}_{0} \rightarrow \mathbb{N}$ be an isomorphism. Then for each $i \in \mathbb{N}$ there is only one pair $(k, n) \in \mathbb{N} \times \mathbb{N}_{0}$ such that $i=f(k, n)$. Now let $D=\left(d_{i j}\right)$ be an infinite matrix whose $i$ th row $D_{i}$ is equal to $C_{k} A^{n}$, where $i=f(k, n)$. If $C$ has only finitely many rows, then the matrix $D$ can be written in the following way:

$$
D=\left(\begin{array}{c}
C \\
C A \\
\vdots
\end{array}\right)
$$

Let $\mathcal{D}(x)=D x, \mathcal{D}: \mathbb{R}^{\mathbb{N}} \rightarrow \mathbb{R}^{\mathbb{N}}$.

Let $e_{i}, i \in \mathbb{N}$ be the infinite row with 1 at the $i$ th position and 0 at other positions.

Proposition 5.6. System $\Lambda$ is observable iff $\forall i \in \mathbb{N} \exists S_{i}=\left\{k_{1}, \ldots, k_{n_{i}}\right\} \subset \mathbb{N}$ $\exists\left\{a_{1}, \ldots, a_{n_{i}}\right\} \subset \mathbb{R}: e_{i}=a_{1} D_{k_{1}}+\cdots+a_{n_{i}} D_{k_{n_{i}}}$.

Remark 5.7. Since the rows of $D$ correspond to derivatives of the output, one can characterize observability as a possibility of computing every state variable as a linear combination of finitely many outputs and their derivatives.

If $C$ has finitely many rows, then for all $0 \leq k<\infty$ the rank of the matrix

$$
\left(\begin{array}{c}
C \\
\vdots \\
C A^{k}
\end{array}\right)
$$

is finite. 
Corollary 5.8. The system $\Lambda$ (with the matrix $C$ of finitely many rows) is observable iff $\forall i \in \mathbb{N} \exists k_{i} \in \mathbb{N}_{0}$ :

$$
\operatorname{rank}\left(\begin{array}{c}
C \\
\vdots \\
C A^{k_{i}}
\end{array}\right)=\operatorname{rank}\left(\begin{array}{c}
C \\
\vdots \\
C A^{k_{i}} \\
e_{i}
\end{array}\right) .
$$

Proposition 5.9. If $\Lambda$ is observable, then $\operatorname{rank} D=\infty$.

Example 5.10. Let $\Lambda$ be the system, on the time scale $\mathbb{T}=\mathbb{Z}$, of the form $x^{\Delta}(t)=A x(t), y(t)=C x(t)$, where $A, C$ are infinite row-finite matrices and $C$ has finitely many rows. Then the necessary and sufficient condition on observability is given by Corollary 5.8. The system $\Lambda$ can be rewritten in the shift form of the discrete-time system: $x(t+1)=(A+I) x(t), y(t)=C(t)$. Then in the condition of observability we should write the matrix $(A+I)$ instead of the matrix $A$. But it does not change this condition because for each $k \in \mathbb{N}$

$$
\operatorname{rank}\left(\begin{array}{c}
C \\
C A \\
\vdots \\
C A^{k}
\end{array}\right)=\operatorname{rank}\left(\begin{array}{c}
C \\
C(A+I) \\
\vdots \\
C(A+I)^{k}
\end{array}\right) .
$$

Remark 5.11. For infinite-dimensional systems observability is usually dual to approximate controllability, which means that the reachable space is dense. Such duality was proved for continuous-time systems described by infinite matrices in $\left[{ }^{6}\right]$. One could try to extend this result to systems on time scales. This would require, however, developing controllability of such systems.

\section{ACKNOWLEDGEMENTS}

D. Mozyrska was supported by the Białystok Technical University grant No. W/WI/7/07, Z. Bartosiewicz by the Białystok Technical University grant No. W/WI/1/07.

\section{REFERENCES}

1. Bartosiewicz, Z. and Pawłuszewicz, E. Realizations of linear control systems on time scales. Control Cybern., 2006, 35, 769-786.

2. Bartosiewicz, Z. and Mozyrska, D. Observability of infinite-dimensional finitely presented discrete-time linear systems. Zesz. Nauk. Politech. Białostockiej. Mat.-Fiz.Chem., 2001, 20, 5-14. 
3. Bartosiewicz, Z. and Mozyrska, D. Observability of row-finite countable systems of linear differential equations. In Proceedings of 16th IFAC Congress, 4-8 July 2005, Prague (Piztek, P., ed.). Elsevier, Oxford, 2006.

4. Curtain, R. F. and Zwart, H. An Introduction to Infinite-Dimensional Linear Systems Theory. Springer-Verlag, New York, 1995.

5. Banach, S. Théorie des opérations linéaires. Warsaw, 1932.

6. Mozyrska, D. and Bartosiewicz, Z. Dualities for linear control differential systems with infinite matrices. Control Cybern., 2006, 36, 887-904.

7. Deimling, R. Ordinary Differential Equations in Banach Spaces. Lecture Notes Math., Vol. 596. Springer-Verlag, 1977.

8. Cooke, R. G. Infinite Matrices and Sequence Spaces. Macmillan, London, 1950.

9. Agarwal, R. P. and Bohner, M. Basic calculus on time scales and some of its applications. Results Math., 1999, 35, 3-22.

10. Bohner, M. and Peterson, A. Dynamic Equations on Time Scales. Birkhauser, Boston, 2001.

11. Bohner, M. and Lutz, A. Asymptotic expansions and analytic dynamic equations. Published online: http://web.umr.edu/ bohner/papers, 2005.

\section{Lineaarsete dünaamiliste lõpmatumõõtmeliste süsteemide klassi jälgitavus ajaskaaladel}

\section{Dorota Mozyrska ja Zbigniew Bartosiewicz}

On uuritud lineaarseid dünaamilisi süsteeme, mille väljund on defineeritud $\mathbb{R}^{\infty}$ lõpmatute jadade ruumis ja mis on esitatud lineaarsete $\Delta$-diferentsiaalvõrrandite lõpmatumõõtmeliste süsteemidega lõplike ridadega maatriksite abil, kus aeg kuulub suvalisse ajaskaalasse. Niisugused süsteemid on varem uuritud diskreetaja ja pideva ajaga $\mathbb{R}^{\infty}$ lõplike ridade süsteemide üldistuseks. On leitud selliste süsteemide jälgitavuse tarvilikud ja piisavad tingimused, milleks on kasutatud formaalseid polünomiaaljadasid ajaskaaladel. 